\title{
5
}

\section{A Simple Model for Estimating Future Channel Migration from Historical Aerial Photographs}

\section{Paul Villard, William Snodgrass and Bishnu Gyawali}

Channel migration can create potential hazards to infrastructure. To minimize the hazard and reduce the need to impact channel form and function through channel hardening, hazard zones are delineated. In areas where infrastructure exists within areas of potential channel migration it is valuable to be able to predict channel migration to develop appropriate management plans. Predicting channel migration is difficult, however, because it is a non-linear process that is a function of the antecedent channel planform and the cumulative interaction of adjacent meanders. This is further complicated by the fact that meanders both migrate and adjust their form over time. To address the complexity a simple process was developed to translate historical meander migration into a series of linear functions that allow prediction of future channel planform. A case study from Sheridan Creek, Mississauga, Ontario is used to illustrate the technique and its potential applications.

Villard, P.V., W. Snodgrass and B. Gyawali. 2009. "A Simple Model for Estimating Future Channel Migration from Historical Aerial Photographs." Journal of Water Management Modeling R235-05. doi: 10.14796/JWMM.R235-05.

(C) CHI 2009 www.chijournal.org ISSN: 2292-6062 (Formerly in Conceptual Modeling of Urban Water Systems. ISBN: 978-0-9808853-2-3)) 


\subsection{Introduction}

Channel migration and erosion creates potential hazards to adjacent infrastructure, particularly for older infrastructure, which in many cases was installed with limited respect for channel dynamics. In many jurisdictions, to minimize the hazard to infrastructure and reduce the need for channel hardening, hazard zones associated with channel migration are delineated. In areas where infrastructure has already been built within zones of potential channel migration it is valuable to be able to estimate rates of migration to evaluate risk and develop appropriate management plans.

Unfortunately predicting channel migration is difficult. Channel planform and migration patterns are a complicated product of local geology, physiography, flow regime, and land cover. Furthermore, the future planform configuration is a function of the antecedent channel planform and the cumulative interactions of adjacent meanders. This is further complicated by the fact that over time individual meanders both migrate and adjust their form.

Numerous approaches have been developed to examine meander migration and planform adjustment of river channels. Phillips and Robert (2007), Stephen et al (2002), Nanson and Hickin (1996), Hooke (1995) have used simple time step comparison and statistical analysis to estimate and describe meander migration and patterns from historical records. Manual overlay of historical maps or aerial photos for assessment of planform adjustment and for estimating meander migration have been developed by Briuad et al. (2001) and Lagasse et al. (2004).

Briuad et al. (2001) superimposed and measured the differences in angle and distance from the centroid of the radius of curvature, which was measured from the inner bank of a given meander bend (Figure 5.1). Extrapolation of the change in these parameters is used to predict future meander position.

Lagasse et al. (2004) described meander migration, similarly, based on the manual overlay from historical aerial photographs. The method measures the migration angle and distance of the centroid of a circle that inscribes the outer bank and the amount of migration of the centroid for each period. Lagasse et al. (2004) proposed two methods for defining the angle of bend migration for the next time step. The first used the previously calculated migration rate and angle for the next time step. This was applied when geomorphic and hydrologic conditions had not changed. The second method used the rate of change between subsequent time steps to adjust the rates of change anticipated during the next time step. 


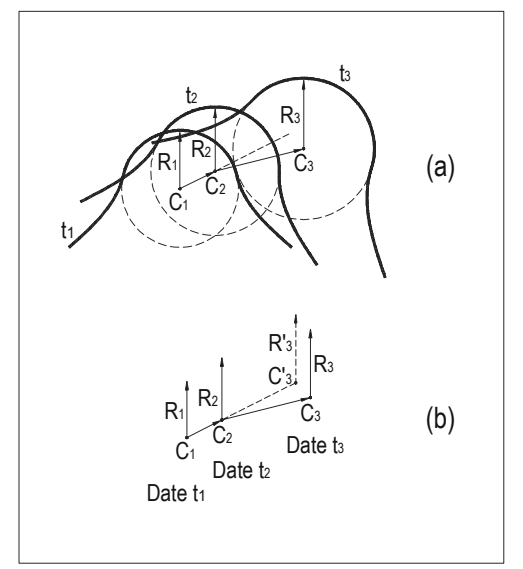

Figure 5.1 Method defined by Briuad et al. (2001) with (a) depicting the change in the meander over time and (b) representing predicted and actual position over time ( $t$ is the time step, $R$ is the radius of curvature, $\mathrm{C}$ is the location of the centroid) (modified from Briuad et al. (2001)).

Both the Briuad et al. (2001) and Lagasse et al. (2004) approaches were developed for large channels, where channel banks are well defined. As such, they do not account for potential adjustment in channel width. Furthermore, using the angle and magnitude of migration of the centroid complicates evaluation of the change in position.

To address these limitations a simple method is outlined in this chapter to translate meander migration and planform adjustment into a series of linear functions related to the radius of curvature and easterly and northerly movement of its centroid. An example of the models application is also described.

\subsection{Methodology}

Figure 5.2 illustrates how the migration of an individual meander can be defined. In Figure 5.2(A) the radius of curvature is defined on the centerline of the channel. A circle defined by the radius of curvature of the meander bend passes through the centerline of the meander at its apex following through the centerline at the two crossover points of the meander. This definition of radius of curvature based on centreline of channel, allows widening to be addressed separately, where it is a significant factor. 
Figure 5.2(B) shows a potential scenario for meander migration. In this case the meander is moving downstream and laterally while elongating. The three plots in Figure 5.2(C) illustrate this motion being separated into three linear functions-based fits related to the radius of curvature and the easterly and northerly movement of its centroid through the three time steps. Although not shown, channel widening, if significant, could be used as a fourth descriptor.

A
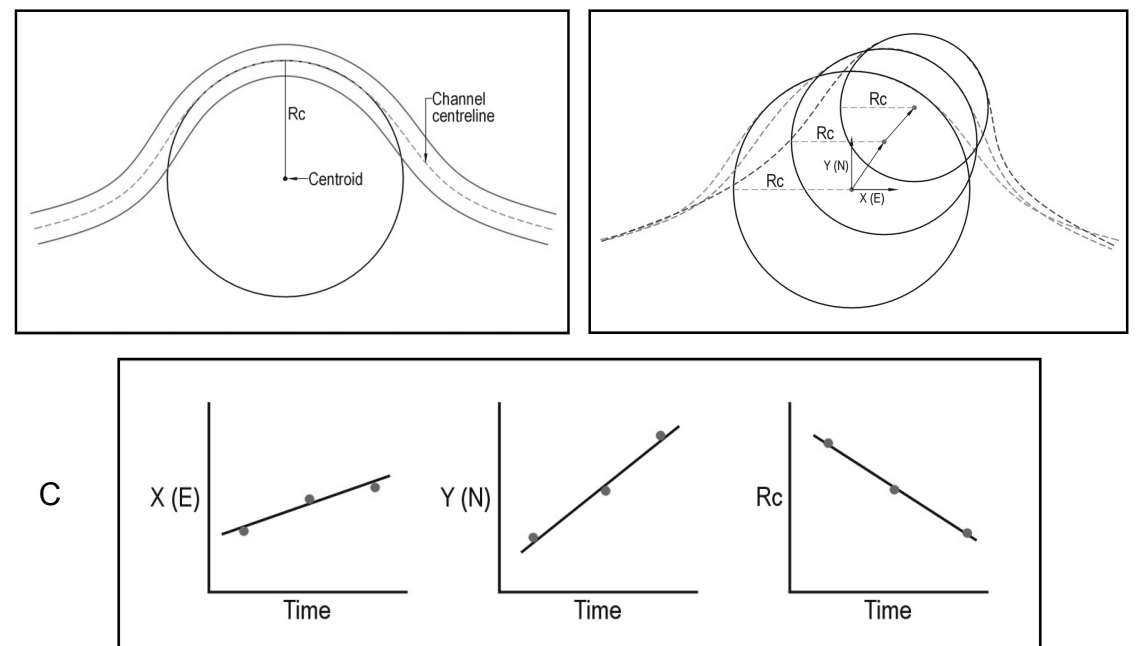

Figure 5.2 Simple visual depiction of the components of the meander migration model.

A linear approach was adopted because it is a simple and stable relation. Application of a linear relation has several assumptions: (i) that there is no systematic change in the hydraulic regime over time, (ii) that local variation in land cover and geology does not significantly impact rates of erosion, and (iii) that there has been no human modification to the channel. With these assumptions in mind, variations from the linear form may indicate situations where these assumptions do not hold. To address this limitation, these types of modeling exercises should include a review of historical and spatial changes in land cover and geology, as well as identifying human modifications to the channel and systematic changes to the hydrological regime. 


\subsection{An Example from Sheridan Creek, Mississauga, Ontario}

To illustrate the application of the model, measurements from Sheridan Creek in Mississauga, Ontario are presented. The Sheridan Creek watershed is within City of Mississauga, Ontario, Canada and drains into Lake Ontario through Rattray Marsh. The section of creek examined is at the downstream extent of the watershed near Rattray Marsh. Aerial photographs from 1944, 1977 and a geo-reference digital aerial photograph from 2005 were selected. The 1944 and 1977 aerial photographs were geo-referenced from the 2005 digital photograph in ArcGIS. Bank lines of the creek were delineated. Six adjacent meanders extending from the mouth of the creek were selected for this example. A circle that defined the radius of curvature of meander bends was delineated for each meander through the three series of aerial photographs. These were delineated from the apex of the centerline of each meander bend following through the centerline of the two crossover points of each meander. The river width was delineated at the apex of the meander bends. The length of radius of curvatures and northing and easting coordinate of the centroid of the circle that defined the radius of curvature were measured.

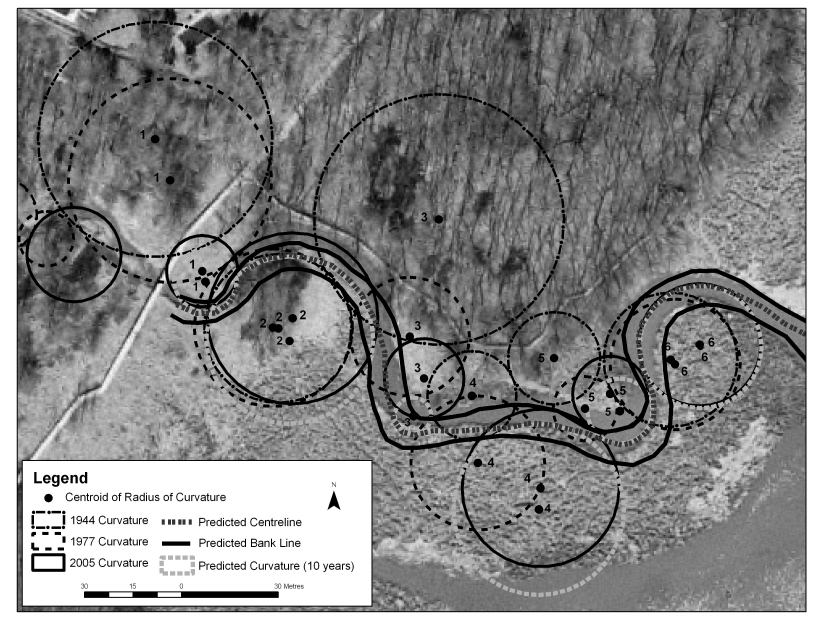

Figure 5.3 Superimposed circles defining the radius curvature of each meander and its centroid for each time step for all six meanders. 
Figure 5.3 illustrates the change in the size and location of the circles that defined the meander position. Each meander is numbered as well as all the past positions associated with the numbered meander. Linear regression was used to define the rate of change with regard to circle position and the radius of curvature for each meander relations. Linear regression was selected as it provided a simple method for defining rate given the very limited number of observations. Although not applied in this example the use of linear regression also provided the opportunity to define confidence bands on the estimates. Figure 5.4 provides an example of the linear relations, in this case, associated with meander 4 .

Although not shown, the results of best-fitted linear regression relations between centroid coordinates and radius of curvatures for each meander allow the pattern of channel evolution to be described and quantified. Examining all the relations the following description is provided. Over the time period examined meander 1 moved to the east and the south (right bank) with a decrease in radius of curvatures. Meander 2 moved to the northeast (left bank) with an increase in radius of curvature. Meander bend 3 migrated to the west and south (right bank) and became narrower. Meander bend 4 migrated to southeast with an increase in the radius of curvature. Meander bend 5 migrated to the southeast (right bank), while meander bend 6 migrated to the northeast (left bank) with a reduction in its radius of curvature.

Meanders 1, 3 and 6 all showed decreasing radius of curvature. Obviously in these cases the radius of curvature cannot drop below zero. The simplest approach is to assume that when the radius of curvature reaches zero the meander ceases to exist. Application of the model to other systems, not presented in this chapter, suggests that narrowing of the meander in many cases is indicative of future loss of the meander through avulsion (meander cutoff) or being dampened out by adjacent meanders.

Although not investigated further in this chapter, by examining the range of radius of curvatures and migration rates observed in the historic record along a given section of channel it may be possible to define a range of possible geometries that could be reasonably anticipated to occur. This could provide system specific cutoffs for the linear models and provide a method to identify anomalous estimates.

Using the linear regression equations the future radius of curvature and the easting and northing coordinate of its centroid can be estimated. In Figure 5.3 a potential future configuration of the planform, for ten-years from the 2005 time step is predicted. This is produced by mapping the position of the centroid of the circle defining the radius of curvature. 
Connecting the centerline of the channel produced by the series of circles delineates the centerline of the new channel planform. The channel width is then superimposed on the centerline of the estimated planform. The advantage of this approach is that it breaks channel planform adjustment into the behaviour and interaction of the individual meanders. Individual meanders can be evaluated to assess immediate risks to adjacent infrastructure or the adjustment of the entire planform of the channel by connecting the trends of adjacent meanders. Although not shown in this chapter, changes in channel widening, which can be a significant factor in bank erosion in small urban streams, can be used as a fourth descriptor. The statistical approach also allows confidence limits to be calculated for the estimates, which will be investigated in the future.
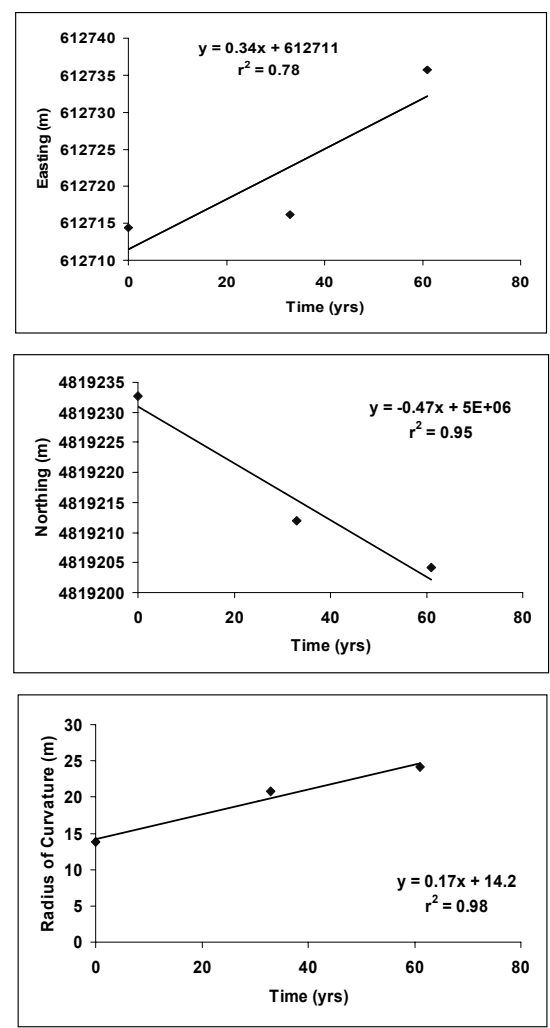

Figure 5.4 Linear regression fits of the change in easting and north coordinates and radius of curvature through the three time steps for meander 4 . 


\subsection{Conclusions}

A simple method was presented to translate historical patterns of meander migration into a series of linear functions that allow estimation of future channel planform. The model predicts meander migration by extrapolating changes in position and radius of curvature through linear regression. The method can be expanded to predict a potential channel planform for a series of meanders by using linear regression confidence limits (to be developed for the estimates, which will be the focus of future work). Using the centre of channel is likely more appropriate on small channels and allows widening in urban systems to be accounted for as a separate variable.

Future areas of investigation and model refinement will likely include assessing whether by defining the range of meander geometries observed in the historic record along a given section of channel, this can be used to provide system specific cutoffs for the linear models and guide identification of anomalous estimates.

\section{References}

Briaud, J-L., Chen, H.-C., Park, S., and Shah, A. (2001) Guidelines for Bridges over Degrading and Migrating Streams, Part 1: Synthesis of Existing Knowledge. Report 2105-2, Cooperative Research Program. Texas transportation Institute, the Texas A\&M University System College Station, Texas Department of Transportation.

Hooke J. M. (1995). River channel adjustment to meander cutoffs on the River Bollin and River Dane, northwest England. Geomorphology 14: 235-253

Lagasse P.F., Spit Z, W.J., Zachmann, D. W. (2004): Handbook for Predicting Stream meander migration National. Cooperative Highway Reach Program (NCHRP) Report 533

Nanson, J,C., and Hickin, E.J. (1996). A statistical analysis of bank erosion and channel migration in western Canada. Geological Society of America Bulletin 97: 497-504.

Phillips R.T.J. and Robert, A. (2007). Hydrologic control of waveforms on small meandering rivers. Earth Surface Processes and Landforms 32: 1533-1546 